\title{
Importância da utilização de ácido alfa lipóico (ALA) e catalase (CAT) no processo de criopreservação de folículos ovarianos pré-antrais, visando reduzir os danos causados pelo estresse oxidativo
}

\section{Importance of the use of alpha lipoic acid (ALA) and catalase (CAT) in the process of criopreservation of pre-ancient ovarian folicles, to reduce the damage caused by oxidative stress}

Luciana Mascena Silva ${ }^{1,2}$. Franciele Osmarini Lunardi1 ${ }^{1}$ Giovanna Quintino Rodrigues ${ }^{1}$. Virginia Cláudia Carneiro Girão ${ }^{2}$. Ana Paula Ribeiro Rodrigues ${ }^{1,2}$.

1 Universidade Estadual do Ceará (UECE), Fortaleza, Ceará, Brasil. 2 Universidade Federal do Ceará (UFC), Fortaleza, Ceará, Brasil.

\section{RESUMO}

A criopreservação de folículos ovarianos pré-antrais é uma perspectiva promissora para a preservação da fertilidade e desenvolvimento de banco de oócitos ou em caso de patologias e terapêuticas que possam comprometer as reservas foliculares. A grande vantagem da criopreservação do tecido ovariano é que independe da idade e fase do ciclo menstrual/estral, além de envolver menos questões éticas e sociais. No entanto, como qualquer exposição de um material biológico a temperaturas extremamente baixas, a criopreservação pode levar a um desequilíbrio na produção de espécies reativas de oxigênio (EROs), podendo causar danos celulares. In vivo, as EROS são equilibradas pelo sistema de defesa de antioxidantes, porém, in vitro, a falta do sistema fisiológico, pode levar ao estresse oxidativo. Portanto, antioxidantes vêm sendo utilizados antes ou após a criopreservação, os quais pode-se destacar o ácido alfa lipóico ou ácido tióctico (ALA) e catalase (CAT). Por tanto, o objetivo desse artigo é auxiliar no entendimento da relevância da criopreservação do tecido ovariano para a preservação da fertilidade das fêmeas, bem como o controle na produção de EROS durante o processo, e a necessidade da suplementação de antioxidantes como o ALA e a CAT.

Palavras-chave: Criopreservação. Espécies reativas de oxigênio. Ácido tióctico. Catalase.

\section{ABSTRACT}

Cryopreservation of preantral ovarian follicles is a promising prospect for the preservation of oocyte fertility and oocyte development or in the case of pathologies and therapeutics that may compromise follicular reserves. The great advantage of cryopreservation of ovarian tissue is that it is independent of the age and stage of the menstrual/estral cycle, and involves fewer ethical and social issues. However, as any exposure of a biological material to extremely low temperatures, cryopreservation can lead to an imbalance in the production of reactive oxygen species (ROS), which can cause cell damage. In vivo, ROS are balanced by the antioxidant defense system, however, in vitro, the lack of physiological system can lead to oxidative stress. Therefore, antioxidants have been used before or after cryopreservation, which can be emphasized alpha lipoic acid or thioctic acid (ALA) and catalase (CAT). Therefore, the objective of this article is to help in understanding the relevance of cryopreservation of ovarian tissue for the preservation of female fertility, as well as the control of ROS production during the process, and the need for supplementation of antioxidants such as ALA and CAT.

Keywords: Cryopreservation. Reactive oxygen species. Thioctic acid. Catalase.

Autor correspondente: Luciana Mascena Silva, Rua Raquel Pires Ribeiro, 43, Mondubim, Fortaleza, Ceará, Brasil. CEP: 60767-255. Telefone: +55 85 99608-0022. E-mail: lumascena2@gmail.com

Conflito de interesses: Não há qualquer conflito de interesses por parte de qualquer um dos autores.

Recebido em: 09 Ago 2018; Revisado em: 28 Mar 2019; Aceito em: 03 Abr 2019. 


\section{INTRODUÇÃO}

Nos últimos anos, os tratamentos utilizados contra o câncer têm resultado em altas taxas de cura e sobrevivência dos pacientes. Contudo, esses tratamentos são gonadotóxicos e levam à infertilidade em quase $100 \%$ dos casos. ${ }^{1}$ As drogas rotineiramente utilizadas, embora altamente citotóxicas, e o transplante de medula óssea, têm permitido a cura de mais de $90 \%$ dos casos. Entretanto, as pacientes que necessitam de quimioterapia e/ou radioterapia para tratamento oncológico, estão propensas a sofrer problemas reprodutivos posteriores, tais como a falência ovariana prematura (FOP). ${ }^{2}$ Desta forma, a criopreservação de tecido ovariano torna-se a principal e mais viável opção para preservar e, consequentemente, regenerar a fertilidade de mulheres que se submeterão a tratamentos contra o câncer. ${ }^{3}$ Para reverter a FOP, o autotransplante de tecido ovariano criopreservado tem sido uma alternativa e relatado com sucesso, com pelo menos 60 crianças saudáveis nascidas ao longo dos últimos 10 anos. $^{4}$

A grande maioria dos relatos de nascimentos após criopreservação e transplante de tecido ovariano tem utilizado o processo de congelação lenta, ${ }^{4}$ porém, alguns autores têm indicado que a vitrificação pode ser melhor que a congelação lenta para a criopreservação de tecido ovariano. ${ }^{5,6}$ Nos últimos anos foram relatados os primeiros nascimentos de humanos a partir de tecido ovariano vitrificado. ${ }^{7}$ Porém, embora tenham sido reportados resultados relevantes, tanto a vitrificação, como a congelação lenta de tecido ovariano, ainda encontram-se em um estágio experimental, provavelmente devido aos protocolos que ainda não estão plenamente estabelecidos.

Portanto, esse artigo de revisão poderá auxiliar no entendimento da importância da criopreservação do tecido ovariano para a preservação da fertilidade e capacidade reprodutiva de fêmeas, bem como para o controle da produção de $\mathrm{ERO}_{\mathrm{S}}$ durante a exposição de materiais biológicos a baixas temperaturas.

\section{CRIOPRESERVAÇÃO DE TECIDO OVARIANO}

A criopreservação de tecido ovariano tem ganhado destaque na reprodução assistida, por salvaguardar um grande número de oócitos imaturos ${ }^{8}$ e preservar a integridade estrutural e funcional do ovário, levando à restauração das funções esteroidogênica e gametogênica. Diferentemente da criopreservação de oócitos maturos ou embriões que necessitam de tratamento hormonal prévio para sua obtenção, a criopreservação do ovário pode ser realizada em qualquer fase da vida da mulher ou da fêmea, o que é considerada uma vantagem dessa técnica. Isso permite que o material genético humano ou de diferentes espécies animais, seja preservado na fase pré-púbere ou impúbere ${ }^{8}$ ou ainda de mulheres que necessitam iniciar imediatamente um tratamento gonadotóxico.

A criopreservação de tecido ovariano pode ser realizada utilizando um método radical de redução da temperatura, na presença de altas concentrações de agentes crioprotetores, sem a formação de cristais de gelo, conhecido como vitrificação. ${ }^{9}$
Diferentemente da congelação lenta, a vitrificação além de evitar a formação intracelular de gelo, ${ }^{10}$ é um método rápido, de fácil execução e de baixo custo. ${ }^{11}$ No entanto, como qualquer exposição de um material biológico a temperaturas extremamente baixas, a vitrificação pode levar a um desequilíbrio na produção de espécies reativas de oxigênio, o que pode induzir a morte celular principalmente por vias apoptóticas mitocondriais.

\section{RADICAIS LIVRES, ESPÉCIES REATIVAS DE OXIGÊNIO E ESTRESSE OXIDATIVO}

Os radicais livres são necessários para as funções corporais fisiológicas, pois estão envolvidos no processo de sinalização intracelular, proliferação, diferenciação e migração celular. ${ }^{12,13}$ São moléculas ou átomos com número ímpar na última camada eletrônica, ${ }^{14}$ ou seja, não pareados, o que determina uma atração para um campo magnético e, algumas vezes torna a substância altamente reativa. ${ }^{15,16}$

Estas moléculas podem ser formadas pela perda (oxidação) ou ganho (redução) de um elétron em reações de óxidoredução. ${ }^{17,18}$ Esses radicais podem ser gerados no citoplasma, mitocôndrias ou na membrana e, seus alvos celulares são as proteínas, lipídios, carboidratos e o DNA. ${ }^{19}$ Eles são capazes de reagir rapidamente com vários compostos celulares, pois possuem uma meia-vida muito curta, variando de poucos minutos a nano segundos. ${ }^{18}$

Apesar de necessários para as funções corporais fisiológicas, em grandes quantidades, os radicais livres são prejudiciais e estão envolvidos na fisiopatologia de várias doenças. ${ }^{14}$

Os radicais livres em que o elétron se encontra desemparelhado em átomo de oxigênio, são classificados como espécies reativas de oxigênio $\left(\mathrm{ERO}_{\mathrm{S}}\right)$, sendo as principais espécies reativas, divididas em dois grupos: o primeiro grupo são os radicais, como a hidroxila $(-\mathrm{HO})$, o superóxido $\left(-\mathrm{O}_{2}\right)$, a peroxila - (ROO) e alcoxila - (RO), e o segundo grupo os não radicalares, como o oxigênio $\left(\mathrm{O}_{2}\right)$, peróxido de hidrogênio $\left(\mathrm{H}_{2} \mathrm{O}_{2}\right)$ e ácido hipocloroso $(\mathrm{HclO}) .^{20,21}$

In vivo, as $\mathrm{ERO}_{\mathrm{s}}$ desempenham um papel importante na proliferação e diferenciação celular ${ }^{20}$ e sua produção ocorre naturalmente na cadeia respiratória mitocondrial, sendo equilibrada pelos sistemas de defesa antioxidantes das células. ${ }^{13}$ No entanto, os elevados níveis de $\mathrm{ERO}_{\mathrm{S}}$ podem induzir a morte celular por vias apoptóticas mitocondriais. ${ }^{22}$

Existem evidências que as $\mathrm{ERO}_{\mathrm{S}}$ são geradas não somente em decorrência do metabolismo celular, mas também por fatores externos, como: concentração de oxigênio, luz e manipulação in vitro. ${ }^{23} \mathrm{O}$ resultado do desequilíbrio entre a produção de $\mathrm{ERO}_{\mathrm{S}} /$ ERNs (Espécies reativas de nitrogênio) e o sistema de defesa antioxidante é conhecido como estresse oxidativo ${ }^{20}$ e esse estresse pode ter impacto negativo sobre os gametas, embriões, estágio pré-natal e pós-natal, ${ }^{24}$ como mostrado na Tabela 1. 
Tabela 1. Impactos do estresse oxidativo sobre os estágios de desenvolvimento.

\begin{tabular}{lllll}
\hline Estágio & Gametas & Embriões & Pré-natal & Pós-natal \\
\hline Impactos & $\begin{array}{l}\text { Alterações dos parâmetros } \\
\text { espermáticos } \\
\text { Decadência genômica }\end{array}$ & $\begin{array}{l}\text { Falha na fertilização e } \\
\text { desenvolvimento }\end{array}$ & Aborto espontâneo & Aborto \\
& $\begin{array}{l}\text { Redução do ATP } \\
\text { Inativação e imaturidade } \\
\text { oocitária }\end{array}$ & Desordem metabólica & & Mutações \\
& & & \\
& Causa aneuploidia & & \\
& Falhas na implantação & & \\
\hline
\end{tabular}

Os riscos de desenvolvimento de estresse oxidativo são maiores in vitro do que in vivo ${ }^{25}$ devido à falta do mecanismo de defesa fisiológica, ausência de antioxidantes naturais e a presença de múltiplas fontes potenciais de $\mathrm{ERO}_{\mathrm{S}}{ }^{26}$ No processo de criopreservação, as altas taxas de resfriamento e aquecimento, bem como a presença de agentes crioprotetores aumentam a produção de $\mathrm{ERO}_{\mathrm{S}}$, levando a alterações no metabolismo oxidativo. ${ }^{27}$ Desta forma, na tentativa de reduzir a formação de $\mathrm{ERO}_{\mathrm{S}}$ in vitro, alguns pesquisadores têm utilizado agentes antioxidantes na solução de criopreservação. ${ }^{28-31}$

\section{ANTIOXIDANTES}

Antioxidantes são substâncias que, mesmo presente em baixas concentrações comparada ao substrato oxidável, podem atrasar ou inibir significativamente as taxas de oxidação desse substrato, ${ }^{32}$ que incluem proteínas, lipídios, carboidratos e DNA, ${ }^{18}$ protegendo assim, as células e seu microambiente dos danos oxidativos. ${ }^{26}$

Os agentes antioxidantes são divididos em enzimáticos e não enzimáticos. Dentre os antioxidantes enzimáticos podemos destacar glutationa (GSH), superóxido dismutase (SOD), catalase (CAT) e glutationa peroxidase (GPx). ${ }^{23}$ No que concerne aos antioxidantes não enzimáticos, destacam-se as vitaminas $\mathrm{C}$ e $\mathrm{E}$, além de diferentes compostos de selênio, ubiquinonas (coenzima Q), ácido úrico e ácido alfa lipoico (ALA). ${ }^{33}$ Os antioxidantes são formados endogenamente ou adquiridos de fontes dietéticas. ${ }^{25}$ No que diz respeito ao sistema reprodutivo, nos machos, os antioxidantes estão presentes nos testículos, epidídimos e plasma seminal. ${ }^{34}$ Nas fêmeas, essas substâncias estão presentes nos ovários, trompas, epitélio do endométrio $^{14}$ e embriões. ${ }^{13}$

No que se refere à reprodução das fêmeas, as ações dos antioxidantes são de fundamental importância, visto que os níveis fisiológicos de EROs quando presentes em excesso, podem comprometer vários aspectos, incluindo a esteroidogênese, foliculogênese, maturação, ovulação e luteólise..$^{13}$ Considerando a presença e importância de agentes antioxidantes no sistema reprodutor, essas substâncias também são frequentemente utilizadas em técnicas de reprodução assistida, como por exemplo, a criopreservação tanto de sêmen ${ }^{35}$ como de oócitos $^{36}$ ou mesmo de tecido ovariano..$^{29-31}$ É importante destacar que as EROs são produzidas nas mitocôndrias, por tanto essa organela merece uma grande atenção especialmente no que se refere aos danos causados na mesma durante o processo de criopreservação.

\section{DANOS MITOCONDRIAIS CAUSADOS PELA CRIOPRESERVAÇÃO}

As mitocôndrias são estruturas delimitadas por duas membranas funcionalmente distintas, uma externa e outra interna, que são altamente dobradas em cristas. Essas duas membranas dão origem a dois compartimentos separados e aquosos, ou seja, o espaço inter membranoso e a matriz intercelular. Essa organela mede entre $0.5-1 \mu \mathrm{m}$ de diâmetro e $7 \mu \mathrm{m}$ de largura, está presente entre 100 a 1000 mitocôndrias por célula e é a única que possui seu próprio genoma, o DNA mitocondrial (mtDNA), organizados na matriz e dependendo das células, pode possuir entre 2 a 10 moléculas de mtDNA. ${ }^{24}$ Essa organela está intrinsecamente envolvida no metabolismo e contribui para várias funções celulares, inclusive a produção de ATP. Além de ter um papel importante durante a apoptose, a homeostase do cálcio, e muitos percursores anabólicos, tais como proteínas, nucleotídeos, e a síntese de esteroides. ${ }^{24}$

A Disfunção mitocondrial tem sido associada a distúrbios, tais como, a infertilidade, envelhecimento e doenças cancerosas. ${ }^{37}$ Em relação aos danos causados nas mitocôndrias pela criopreservação, uma das consequências inevitáveis, é o choque térmico, o qual pode afetar a organização celular ou inativar os sistemas enzimáticos, com consequentes mudanças nas propriedades estruturais e funcionais das células. Nessas condições físicas e químicas, a tolerância do tecido ao estresse é reduzida, o qual pode resultar no metabolismo aeróbico do tecido com consequente disfunção mitocondrial..$^{38}$ Essas alterações são resultantes das forças osmóticas geradas durante o congelamento e aquecimento, afetando as atividades, principalmente citoplasmáticas, tais como a função mitocondrial, metabolismo e vias de sinalização intracelulares. ${ }^{39}$

\section{Ácido alfa lipoico (ALA)}

O ALA está presente nas mitocôndrias e funciona como um cofator de complexos multienzimáticos. Esse antioxidante reage com as EROs, como: radicais superóxido, radicais hidroxila, ácido hipocloroso, radicais peroxil e oxigênio singlete. Essa substância também protege as membranas ao 
interagir com a vitamina $\mathrm{C}$ e a glutationa que por sua vez pode reciclar a vitamina $\mathrm{E}$. De fato, foi observado que o ALA impediu os sintomas de escorbuto em cobaias com deficiência de vitamina $\mathrm{C}$, bem como, preveniu os sintomas de deficiência de vitamina $\mathrm{E}^{40}$

O ALA tem influência sobre o fator de crescimento do nervo (NGF), induzindo a expressão do gene superóxido dismutase, que resulta em aumento do antioxidante SOD, além de aumentar a atividade da CAT e GPx, ${ }^{41}$ duas enzimas fundamentais que podem ser utilizadas como biomarcadores do estresse oxidativo. ${ }^{42}$ Algumas evidências também indicam que o ALA pode suprimir a morte celular através da produção de glutationa ${ }^{43}$ e inibir o fator de necrose tumoral alfa (TNF- $\alpha$ ) induzido pela produção de espécies reativas de oxigênio. ${ }^{44}$

\section{Utilização de ALA no cultivo in vitro de folículo pré-antrais criopreservados}

Em relação ao cultivo in vitro de folículos pré-antrais isolados, o ALA mostrou-se um antioxidante potente sobre a sua capacidade de desenvolvimento desses folículos em murinos (TALEBI et al., 2012). A adição do ALA no cultivo in vitro de 12 dias de folículos pré-antrais frescos e vitrificados de camundongos, reduziu os níveis de $\mathrm{ERO}_{\mathrm{S}}$ e aumentou a capacidade oxidativa total, melhorando assim as taxas de sobrevivência e competência para o desenvolvimento desses folículos. ${ }^{45}$

\section{Utilização de ALA na solução de vitrificação de folículo pré- antrais criopreservados}

Na criopreservação, até o momento, foi somente utilizada na solução de vitrificação de tecido ovariano ovino, o qual ALA na concentração de $100 \mu \mathrm{M} / \mathrm{mL}$ de ALA preservou a morfologia, promoveu o desenvolvimento, causou menos danos no DNA dos folículos pré-antrais e manteve os níveis de ROS no meio de incubação e atividade mitocondrial inalterados quando comparado ao controle. ${ }^{31}$

\section{Catalase (CAT)}

A CAT é uma enzima que, em mamíferos, localiza-se no peroxissomo e, uma de suas funções é catalisar o peróxido de hidrogênio $\left(\mathrm{H}_{2} \mathrm{O}_{2}\right.$ em oxigênio molecular e água $\left(2 \mathrm{H}_{2} \mathrm{O}_{2}\right.$ $\rightarrow \mathrm{O}_{2}+2 \mathrm{H}_{2} \mathrm{O}$ ), diminuindo o risco de formação do radical hidroxila, ${ }^{33}$ que pode levar à inativação ou mutação do DNA e inativação de várias proteínas de membrana. ${ }^{32}$

\section{REFERÊNCIAS}

1. Donnez J, Dolmans MM, Pellicer A, Diaz-Garcia C, Serrano MS, Schmidt KT, et al. Restoration of ovarian activity and pregnancy after transplantation of cryopreserved ovarian tissue: a review of 60 cases of reimplantation. Fertil Steril. 2013;99:1503-13.

2. Meirow D. Fertility preservation in cancer patients using stored ovarian tissue: clinical aspects. Curr Opin Endocrinol Diabetes Obes. 2008;15(6):536-47.
A peroxidação lipídica ou lipoperoxidação é a incorporação de um oxigênio molecular (radical livre) sobre os ácidos graxos da membrana celular, levando à destruição de sua estrutura, perda das trocas metabólicas e, em última condição, à morte celular. A peroxidação lipídica parece ser o principal dano causado às membranas ${ }^{46}$ e evidências sugerem que a CAT é capaz de reduzir esses danos..$^{29,30}$

\section{Utilização da CAT na criopreservação de oócitos e folículos pré-antrais}

A Catalse foi utilizada em combinação com superóxido desmutase (SOD) na solução de congelação lenta de óocitos de camundongos, e demonstrou-se que essa associação pode proteger contra um desequilíbrio no sistema de reduçãooxidação. ${ }^{36}$

Durante o congelamento lento de tecido ovariano caprino, a suplementação de CAT na concentração de 20UI/ml na solução de criopreservação conservou a viabilidade folicular e reduziu a peroxidação lipídica em relação ao controle. ${ }^{29}$

Nesta mesma espécie, a ausência de CAT nas soluções de vitrificação resultou em uma maior produção de EROs comparado ao tecido ovariano fresco ou não vitrificado, propondo que a CAT controla a produção de espécies reativas de oxigênio. ${ }^{30}$ Utilizado também na solução de vitrificação de tecido ovariano ovino, a CAT foi menos eficiente do que o ALA em relação a preservação da morfologia e ao desenvolvimento folicular. ${ }^{31}$

\section{CONSIDERAÇÕES FINAIS}

Um dos aspectos que necessitam ser aprimorados nos protocolos de criopreservação é o controle da produção de espécies reativas de oxigênio, o qual é muito comum durante esse processo e geralmente causado pela redução de temperatura, utilização de crioprotetores, estresse osmótico e toxicidade. Portanto, uma das alternativas é a suplementação das soluções de criopreservação com antioxidantes, visando reduzir as crioinjúrias, o que pode ser considerado de extrema relevância para a pesquisa e consequente avanço da criobiologia. Além disso, esse protocolo pode servir para a aplicabilidade prática do processo de criopreservação do material genético de fêmeas. Visto a importância da utilização dos antioxidantes ALA e CAT nas soluções de criopreservação de folículos pré-antrais, torna-se necessário realizar mais estudos no sentido de aprimorar a técnica de criopreservação.

3. Dolmans MM, Jadoul P, Gilliaux S, Amorim CA, Luyckx V, Squifflet J, et al. Review of 15 years of ovarian tissue bank activities. J Assist Reprod Genet. 2013;30:305-14.

4. Donnez J, Dolmans MM. Transplantation of ovarian tissue. Best Pract Res Clin Obstet Gynaecol. 2014;28(8):1188-97.

5. Bandeira FT, Carvalho AA, Castro SV, Lima LF, Viana DA, 
Evangelista JS, et al. Two methods of vitrification followed by in vitro culture of the ovine ovary: evaluation of the follicular development and ovarian extracellular matrix. Reprod Domest Anim. 2015;50(2):177-85.

6. Sanfilippo S, Canis M, Smitz J, Sion B, Darcha C, Janny $\mathrm{L}$, et al. Vitrification of human ovarian tissue: a practical and relevant alternative to slow freezing. Reprod Biol Endocrinol. 2015;13:67.

7. Kawamura K, Cheng Y, Suzuki N, Deguchi M, Sato Y, Takae $\mathrm{S}$, et al. Hippo signaling disruption and Akt stimulation of ovarian follicles for infertility treatment. Proc Natl Acad Sci U S A. 2013;110(43):17474-9.

8. Dela Pena EC, Takahashi Y, Katagiri S, Atabay EC, Nagano M. Birth of pups after transfer of mouse embryos derived from vitrified preantral follicles. Reproduction. 2002;123(4):593-600.

9. Wowk B. Thermodynamic aspects of vitrification. Cryobiology. 2010;60(1):11-22.

10. Rubinsky B. Principles of low temperature cell preservation. Heart Fail Rev. 2003;8(3):277-84

11. Yeoman RR, Wolf DP, Lee DM. Coculture of monkey ovarian tissue increases survival after vitrification and slow-rate freezing. Fertil Steril. 2005;83(4):1248-54.

12. Ford WC. Reactive oxygen species and sperm. Hum Fertil. 2001;4(2):77-8.

13. Agarwal AK, Nallella P, Allamaneni S, Said TM. Role of antioxidants in treatment of male infertility: an overview of the literature. Reprod Biomed Online. 2004;8(6):616-27.

14. Rakhit $\mathrm{M}$, it $\mathrm{M}$, GokulSR, Agarwal A, Plessis SS. Antioxidantstrategies to overcome OS in IVF-Embryo transfer. In: Agarwal A, Aziz N, Rizk B (eds). Studies on women'shealth. Oxidative stress in applied basic research and clinical practice. Totowa:Humana Press; 2013. p. 237-62.

15. Halliwell B, Gutteridge JM, Cross CE. Free radicals, antioxidants, and human disease: where are we now? J Lab Clin Med. 1992;119(6):598-620.

16. Perrota V, Shinaider A. Radicais livres de oxigênio: importância na fisiopatologia das lesões isquêmicas viscerais. Ann Acad Med. 1992;152:22-7.

17. Ferreira AL, Matsubara LS. Radicais livres: conceitos, doenças relacionadas, sistema de defesa e estresse oxidativo. Rev Assoc Med Bras. 1997;43(1):61-8.

18. Vannucchi H, Moreira EA, Cunha DF, Junqueira-Franco MV, Bernardes MM, et al. Papel dos nutrientes na peroxidação lipídica e no sistema de defesa antioxidante. Medicina (Ribeirao Preto). 1998;31(1):31-44.

19. Mirzaei H, Regnier F. Protein: protein aggregation induced by protein oxidation. J Chromatogr B Analyt Technol Biomed Life Sci. 2008;873(1):8-14.

20. Droge W. Free radicals in the physiological control of cell function. Physiol Rev. 2002;82(1):47-95.

21. Barreiros AL, David JM, David JP. Estresse oxidativo: relação entre geração de espécies reativas e defesa do organismo. Quím Nova. 2006;29(1):113-23.

22. Chandra J, Samali A, Orrenius S. Triggering and modulation of apoptosis by oxidative stress. Free Radic Biol Med. 2000;29(34):323-33.

23. Combelles CM, Gupta S, Agarwal A. Could oxidative stress influence the in-vitro maturation of oocytes? Reprod Biomed Online. 2009;18(6):864-80.

24. Benkhalifa M, Ferreira YJ, Chahine H, Louanjli N, Miron P, Merviel P, et al. Mitochondria: participation to infertility as source of energy and cause of senescence. Int J Biochem Cell Biol. 2014;55:604.

25. Amaral S, Amaral A, Ramalho-Santos J. Aging and male reproductive function: a mitochondrial perspective. Front Biosci (Schol Ed). 2013;5:181-97.

26. Gupta S, Malhotra N, Sharma D, Chandra A, Agarwal A. Oxidative stress and its role in female infertility and assisted reproduction: clinical implications. Int J Fertil Steril. 2009;2:147-64.

27. Lampiao F. Free radicals generation in an in vitro fertilization setting and how to minimize them. Int J Fertil Steril. 2012;1(3):2934.

28. Dowling DK, Simmons LW. Reactive oxygen species as universal constraints in life-history evolution. Proc Biol Sci. 2009;276(1663):1737-45.

29. Tatone C, Di Emidio, Vento M, Ciriminna R, Artini PG. Cryopreservation and oxidative stress in reproductive cells. Gynecol Endocrinol. 2010;26:563-7.

30. Luz HK, Santos RR, Wanderley LS, Faustino LR, Silva CM, Carvalho AA, et al. Catalase prevents lipid peroxidation and enhances survival of caprine preantral follicles cryopreservedin a 1,2-propanediol-freezing medium. Biopreserv Biobank. 2012;10(4).

31. Carvalho AA, Faustino LR, Silva CM, Castro SV, Lobo CH, Santos FW, et al. Catalase addition to vitrification solutions maintains goat ovarian preantral follicles stability. Res Vet Sci. 2014;97:140-7.

32. Halliwell B, Gutteridge JM. Free radicals in biology and medicine. 2. ed. Oxford: Clarendon Press; 1989.

33. Nordberg J, Arner ES. Reactive oxygen species, antioxidants, and the mammalian thioredoxin system. Free Radic Biol Med. 2001;31(11):1287-1312.

34. Tremellen K. Oxidative stress and male infertility-a clinical perspective. Hum Reprod Update. 2008;14(3):243-58.

35. Shafiei M, Forouzanfar M, Morteza HS, Hossein MN. The effect of superoxide dismutase mimetic and catalase on the quality of postthawed goat sêmen. Theriogenology. 2015;83(8):1321-27.

36. Dinara S, Sengoku K, Tamate K, Horikawa M, Ishikawa M. Effects of supplementation with free radical scavengers on the survival and fertilization rates of mouse cryopreserved oocytes. Hum Reprod. 2001;16:1976-81.

37. Amaral S, Amaral A, Ramalho-Santos J. Aging and male reproductive function: a mitochondrial perspective. Front Biosci. 2013;5:181-97. 
38. Fabbri R, Vicenti R, Martino NA, Dell'Aquila ME, Pasquinelli G, Macciocca M, et al. Confocal laser scanning microscopy analysis of bioenergetic potential and oxidative stress in fresh and frozenthawed human ovarian tissue from oncologic patients. Fertil Steril. 2014;101(3):795-804.

39. Salehnia M, Töhönen V, Zavareh S, Inzunza J. Does cryopreservation of ovarian tissue affect the distribution and function of germinal vesicle oocytes mitochondria? Biomed Res Int. 2013;2013:8.

40. Packer L, Witt E, Tritschler J. Alpha-lipoic acid as a biological antioxidant. Free Radic Biol Med. 1995; 19(2):227-50.

41. Akpinar D, Yargicoglu P, Derin N, Aliciguzel Y, Agar A. The effect of lipoic acid on antioxidant status and lipid peroxidation in rats exposed to chronic restraint stress. Physiol Res. 2008;57(6):893901.
42. Luberda $Z$. The role of glutathione in mammalian gametes. Reprod Biol. 2005;5(1):5-17.

43. Voloboueva LA, Liu J, Suh JH, Ames BN, Miller SS. (R)-alphalipoic acid protects retinal pigment epithelial cells from oxidative damage. Invest Ophthalmol Vis Sci. 2005;46(11):4302-10.

44. Byun CH, Koh JM, Kim DK, Park SI, Lee KU, Kim GS. Alphalipoic acid inhibits TNF-alpha-induced apoptosis in human bone marrow stromal cells. J Bone Miner Res. 2005;20(7):1125-35.

45. Hatami S, Zavareh S, Salehnia M, Lashkarbolouki T, Ghorbanian MT, Karimi I. The impact of alpha lipoic acid on developmental competence of mouse vitrified pre-antral follicles in comparison to those isolated from vitrified ovaries. Iran Biomed J. 2014;12(1):57-64.

46. Srivastava, S, Kumar, S. Incorporation of Ascorbic Acid, Caffeine and Chloroquine Diphosphate in Dilutor Improves Structural and Functional Status of Frozen Semen. Scientific Research. 2014;1(1):1-12.

\section{Como citar:}

Silva LM, Lunardi FO, Rodrigues GQ, Girão VC, Rodrigues AP. Importância da utilização de ácido alfa lipóico (ALA) e catalase (CAT) no processo de criopreservação de folículos ovarianos pré-antrais, visando reduzir os danos causados pelo estresse oxidativo. Rev Med UFC. 2020 jan-mar;60(1):41-46. 